\title{
Alternativa con gel de Papaver somniferum (amapola) y Cannabis sativa (marihuana), como tratamiento de artritis reumatoide
}

\author{
Ana R Ramírez Jiménez,* Adrián Ruiz, Olvera,* Virginia Melo Ruiz, ** Maritza García***
}

\begin{abstract}
RESUMEN
En la elaboración del presente producto farmacéutico (una formulación galénica entre el gel y la emulsión fluida), se utilizaron dos principios activos naturales considerados psicotrópicos o narcóticos: extracto alcohólico de amapola (Papaver somniferum) y extracto alcohólico de marihuana (Cannabis sativa); éstos tienen actividad biológica conocida dentro del organismo. Son excelentes analgésicos para pacientes con cáncer terminal pero también tienen propiedades antieméticas, estimulantes del apetito, relajantes oculares, bronquíticos, ansiolíticos. Se conoce que para tener una liberación prolongada de un principio activo es muy útil el uso de liposomas; por esta razón se utilizaron liposomas de extracto alcohólico de amapola, considerando que esta planta tiene mejores propiedades analgésicas que la marihuana, por lo cual el extracto de esta última integra un $40 \%$ de la formulación. Los resultados obtenidos después de la elaboración del producto fueron favorables en lo relacionado al control de calidad. El presente trabajo comprende un gel, para el uso tópico; esta composición farmacéutica es un reservorio de ingredientes activos en un sistema de liberación transdérmico, además tiene una notable estabilidad y características orgalépticas óptimas.
\end{abstract}

Palabras clave: Liposomas, gel, Cannabis sativa, Papaver somniferum.

\section{Alternative gel Papaver somniferum (poppy) and Cannabis sativa (marihuana), as treatment of rheumatoid arthritis}

\begin{abstract}
The development of this pharmaceutical product is a pharmaceutical formulation between gel and fluid emulsion, which used two natural active ingredients considered psychotropic or narcotic, alcoholic extract of poppy (Papaver somniferum) and alcoholic extract of marijuana (Cannabis sativa), they have known biological activity within the body. They are excellent analgesics for terminal cancer patients but also have antiemetic properties, stimulating appetite, soothing eye, bronchitis, anxiety. It is known that for a sustained release of active principle is very useful the use of liposomes; for this reason, liposomes of alcoholic extract of poppy considering that this plant has better analgesic properties of marijuana for which the latter extract includes $40 \%$ of the formulation. The results obtained after processing of the product were favorable in relation to quality control. The work is a gel for topical use, this pharmaceutical composition is a reservoir of active ingredients in a transdermal delivery system, has a remarkable stability and optimal orgaleptics characteristics.
\end{abstract}

Key words: Liposomes, gel, Cannabis sativa, Papaver somniferum.

\footnotetext{
*Universidad Autónoma Metropolitana-Xochimilco.

**Maestría en Biología.

**** Maestra, Doctora en enfermería.
}

Correspondencia: Ana R. Ramírez Jiménez. Calzada del Hueso Núm. 1100, Col. Villa Quietud, Delegación Coyoacán, 04960,

México, D.F. Tel. 5483-7000

Este artículo puede ser consultado en versión completa en http://www.medigraphic.com/enfermerianeurologica 


\section{INTRODUCCIÓN}

$\mathrm{L}$ a artritis reumatoide es una enfermedad sistémica crónica de causa desconocida que afecta de modo principal las glándulas sinoviales de múltiples articulaciones. Esta enfermedad tiene una gran cantidad de manifestaciones, tanto articulares como extraarticulares, que suelen iniciar entre los 20 y 40 años, aunque puede ser a cualquier edad. La susceptibilidad de la artritis reumatoide está determinada de manera genética. Esta enfermedad está ampliamente distribuida en el mundo. La prevalencia de AR es aproximadamente de $1 \%$ de la población (oscila entre el 0.3 y $2 \%$ ). Las mujeres se afectan aproximadamente en una proporción tres veces mayor que los varones. La prevalencia aumenta con la edad, manifestándose una incidencia de la AR en las mujeres de 60 a 64 años en proporción más de seis veces mayor que las de 18 a $29 .{ }^{4}$

Los estudios muestran una predisposición genética. Los factores de riesgo no explican, en su totalidad, la incidencia de AR, lo que sugiere la participación de los factores ambientales en la etiología. El clima y la urbanización poseen gran impacto en la incidencia y gravedad de AR en grupos con una base genética similar. ${ }^{8}$

La terapia farmacológica empleada para la $\mathrm{AR}$ es en gran medida paliativa, y está destinada tanto al alivio del dolor como a la disminución de la inflamación de la articulación o articulaciones implicadas; sin embargo, hasta el momento no hay ninguna medicación curativa.

Aunque existe una amplia gama de manifestaciones, la alteración característica de la $\mathrm{AR}$ es una sinovitis inflamatoria persistente que afecta habitualmente a las articulaciones periféricas con una distribución simétrica. El signo clave de la enfermedad es el potencial de la inflamación sinovial para producir una destrucción del cartílago con erosiones óseas y deformidades articulares en fases posteriores. A pesar de su potencial destructor, la evolución de la AR puede ser muy variable..$^{1,5,9,8}$

La AR presenta una distribución universal, estimándose una prevalencia mundial en torno al $1 \%$ y una incidencia aproximada de 0.5 casos anuales por 1,000 habitantes. Esta enfermedad conlleva además un importante costo económico, estimándose que supera los 1.2 millones de euros anuales, de los cuales unos 400 millones corresponderían al gasto sanitario. Las tasas más elevadas de prevalencia están entre los 40-60 años, con mayor frecuencia en mujeres, aunque esta proporción no es tan acusada en el AR del anciano. ${ }^{4}$ Se asocia a diversos factores de riesgo, entre los que cabe destacar la edad avanzada ( $<65$ años) la obesidad y los traumatismos y sobrecarga continuados de las matriculaciones (por ejercicio físico intenso, determinados trabajos, etc.). Se manifiesta principalmente por la presencia de un dolor "sordo" en la articulaciones afectadas, que inicialmente puede ser leve e intermitente, pero que puede llegar a se muy intenso y persistente; el dolor se asocia a otros síntomas como inflamación, rigidez y reducción de la movilidad y funcionalidad de los pacientes. ${ }^{3}$

$\mathrm{El}$ proceso inflamatorio pone en peligro tendones, ligamentos, aponeurosis, músculo y huesos. Los mediadores de la inflamación pueden trasladar el trastorno a diferentes estructuras orgánicas. ${ }^{1}$

Algunos pacientes pueden presentar únicamente un proceso inflamatorio en articulaciones pequeñas, de breve duración y con lesiones articulares mínimas, mientras que otros presentan una poliartritis progresiva en imparable evolución hacia la aparición de deformidades articulares importantes. ${ }^{9}$

\section{Sistemas liposomales}

Los liposomas son estructuras macromoleculares intensamente estudiadas y caracterizadas en los últimos años, capaces de atrapar fármacos y servir como vehículos de transporte de los mismos. Estas estructuras fueron producidas por primera vez en 1961 y se estudiaron inicialmente como modelos de membranas celulares y para estudiar el transporte de iones a través de membranas biológicas. ${ }^{6}$

Posteriormente comenzaron a ser evaluadas como medios de transporte de drogas y en 1988 se registra en Suiza el primer preparado que contenía liposomas: el econazol al $1 \%$ (Pevaryl Lipogel de Cilag). ${ }^{2}$ Se consideran sistemas de liberación debido a su capacidad para transformar sustancias activas hidrófilas en el comportamiento acuoso y sustancias activas hidrófobas entre los fosfolípidos de la capa biomolecular; su composición es semejante a la de las membranas celulares. ${ }^{12} \mathrm{Al}$ comprobarse el aumento de la biodisponibilidad biofásica del fármaco por su incorporación a liposomas, la aplicación terapéutica de estos sistemas ha ido desarrollándose paulatinamente?

\section{Opioides}

Teniendo en cuenta que existe un cúmulo de enfermedades no oncológicas que, debido a su desarrollo progresivo e inexorable, conllevan la incurabilidad (p. ej. enfermedades neurológicas, cardiológicas, respiratorias, etc.), y son generadoras de dolor, la aplicación de las habilidades de los cuidados paliativos y de la medicina del dolor, resulta esencial para el cuidado y el alivio en estos pacientes. Algunos síntomas entre estas dos poblaciones son similares. Usualmente, en el paciente con cáncer el dolor es más intenso; en cambio, en el paciente con enfermedad dolorosa crónica no oncológica, el dolor tiende a ser mucho más prolongado, generando mayor sufrimiento. ${ }^{9}$ 
En este sentido, la utilización de opioides tiene un lugar preponderante no solamente como excelentes analgésicos, sino por su alta eficacia en el tratamiento de otros síntomas (disnea nocturna, tos, etc.).

Se ha demostrado fehacientemente que los opioides pueden estimular el haz hipotálamo-hipofisario-suprarrenal, favoreciendo las propiedades antiinflamatorias de este eje, tan bien como el sistema de citoquinas, fundamentalmente en la artritis reumatoide. ${ }^{16,14}$

El objetivo principal de esta investigación consiste en el desarrollo de una formulación alternativa para la administración de Papaver somniferum (amapola) y Cannabis (marihuana) en condiciones de eficacia y seguridad, mediante su asociación química a un sistema transportador (liposomas), la cual tendrá una presentación farmacéutica en gel.

Por las características propias de la amapola así como de la marihuana, el producto resulta una propuesta para aliviar dolores reumáticos y musculares.

\section{MATERIAL Y MÉTODOS}

Se utilizó una infusión alcohólica de Papaver somniferum (amapola) y de Cannabis sativa (marihuana) en una presentación de $250 \mathrm{~mL}$. Ambos concentrados fueron obtenidos en Chapingo.

Papaver somniferum (amapola). Esta planta que posee flores de color blanco, rojas y púrpuras, se cultiva por su látex (el opio) y fue obtenida en una infusión alcohólica de $250 \mathrm{~mL}$ en Chapingo. Debido a que el opio posee más de 70 alcaloides y aproximadamente 25 componentes activos, permite que sus indicaciones medicinales y la de sus derivados sean múltiples fomentando así que la industria farmacéutica elabore conocidos anestésicos, antiespasmódicos, antitusígenos, eutimizantes, ansiolíticos, hipnóticos, sedantes, oncológicos (en la medicina paliativa mitigantes del dolor fuerte en las afecciones principalmente cancerosas) y en el tratamiento de la disnea (sofocación).$^{15}$ Los alcaloides más importantes son, por su uso: morfina, que se considera el principal alcaloide del opio ya que posee propiedades analgésicas, hipnóticas y contribuye a contener las hemorragias; morfina destinada al consumo para fines terapéuticos, así como codeína, papaverina, noscarina, narceína y narcotina. También se obtiene la tebaína, analgésico obtenido de la amapola de la tebaida y de la cual se obtiene también la codeína (metilmorfina). ${ }^{13}$

Cannabis sativa (marihuana). Posee 426 compuestos químicos, 60 de los cuales son sus componentes activos producidos principalmente en las flores. De éstos, hay 9 cuyos ingredientes psicoactivos, de la familia $\mathrm{TCH}$, conocidos como cannabinoides. ${ }^{1}$

Entre los cannabinoides, considerados terapéuticamente más eficaces, figuran el THC (9 Delta tetrahidrocannabinol) y el CBD (cannabidiol), motivo por el cual han sido los más estudiados. La marihuana natural es consumida en medicina de múltiples maneras: Inhalada o fumada (contra el asma, para mejorar la visión nocturna, la opresión, la sofocación, el insomnio, la tisis laríngea, la ronquera, la extinción de la voz, las neuralgias faciales y como relajante); en infusiones (para combatir las náuseas y otras molestias causadas por los efectos secundarios de la quimioterapia en pacientes con cáncer), aplicada (para combatir el dolor). Terapéuticamente se aconsejó para tratamientos de insomnio y como sedante para el dolor (en artritis, reumatismo, lesiones de médula, paraplejia y tetraplejía). También se prescribió para terapias de patologías nerviosas (tranquilizante para maníaco-depresivos, antihistérico, vómito nervioso, tos nerviosa, epilepsia). ${ }^{3}$ En México, la Secretaría de Salud clasifica a cannabis, resina de cannabis, extractos y tinturas de cannabis en la lista amarilla de estupefacientes sometidos a fiscalización nacional. ${ }^{10}$

Durante un periodo de dos semanas se elaboró un gel de

Papaver somniferum con Cannabis sativa como principios activos. Durante los primeros días se obtuvieron liposomas hidrosolubles y liposolubles de alcohol de Papaver somniferum; dentro de los excipientes de dichos liposomas, se ocupó un disolvente que es el etanol; con fines de aumentar el rendimiento, el proceso de elaboración se llevó por duplicado, sustituyendo en una de las formulaciones el etanol por cloroformo; se analizó microscópicamente, para observar el rendimiento de los liposomas; posteriormente, se procedió a la elaboración del gel como presentación final: como en el caso anterior, se sustituyó uno de los excipientes para el gel con liposomas hidrosolubles; se ocuparon como conservadores el metilparaben y el propilparaben; para el gel con liposomas liposolubles se ocupó el benzoato de sodio, el cual cumple con la misma función; definido esto, se agregó en alícuotas de $10 \mathrm{~mL}$ el alcohol de Cannabis sativa y hasta el final y siguiendo la misma metodología se agregaron los liposomas respectivamente; el producto final también se observó en el microscopio para verificar la existencia de los liposomas y finalmente proceder a su envasado y etiquetado. ${ }^{13}$

\section{PRUEBAS}

\section{Microscopia óptica}

Durante el proceso de elaboración de los liposomas y el gel como presentación final se deben realizar las observaciones a microscopio para observar el rendimiento de los liposomas. ${ }^{8,11}$

\section{Pruebas organolépticas}

$\mathrm{Al}$ producto final se debe aplicar una porción del gel en la mano para sentir la consistencia del gel y verificar si deja 
residuos, y también observar la formación de grumos dentro del gel y también el olor del mismo. ${ }^{11,8}$

\section{RESULTADOS Y DISCUSIÓN}

Durante el análisis microscópico de la elaboración de los liposomas, se observó un mayor rendimiento de los liposomas liposolubles, con un conteo por cuadrante de 15 liposomas por 2 liposomas de los hidrosolubles; al igual que el proceso de elaboración, fue más rápido en el primer caso; en general, el rendimiento fue bajo entre los 2 liposomas, tanto liposolubles como hidrosolubles, pero fue óptima su incorporación al gel ya que se consideraba la posibilidad de que durante el proceso de agitado se perdieran liposomas, lo cual no fue así, ya que se mantuvo dicho conteo.

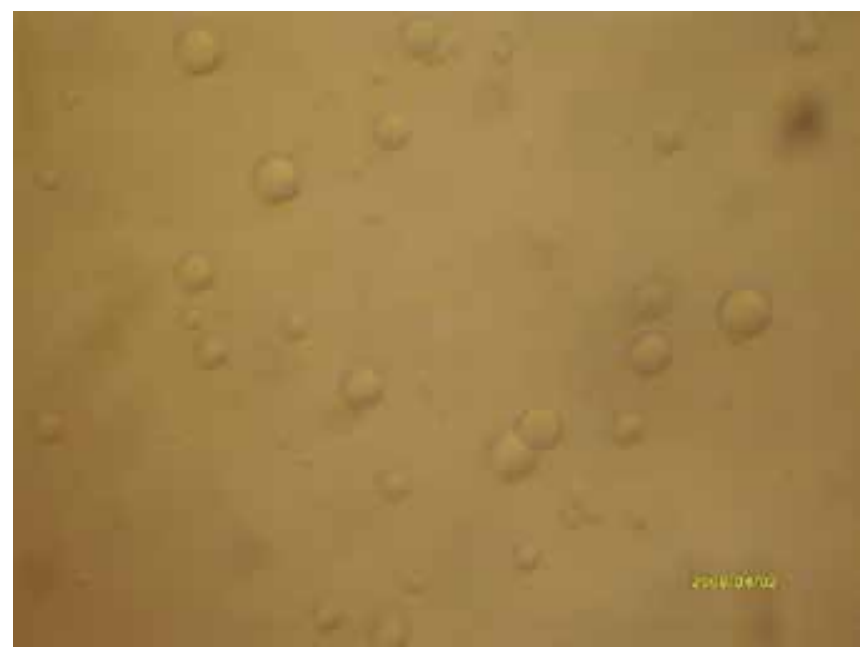

Figura 1. Liposomas liposolubles.

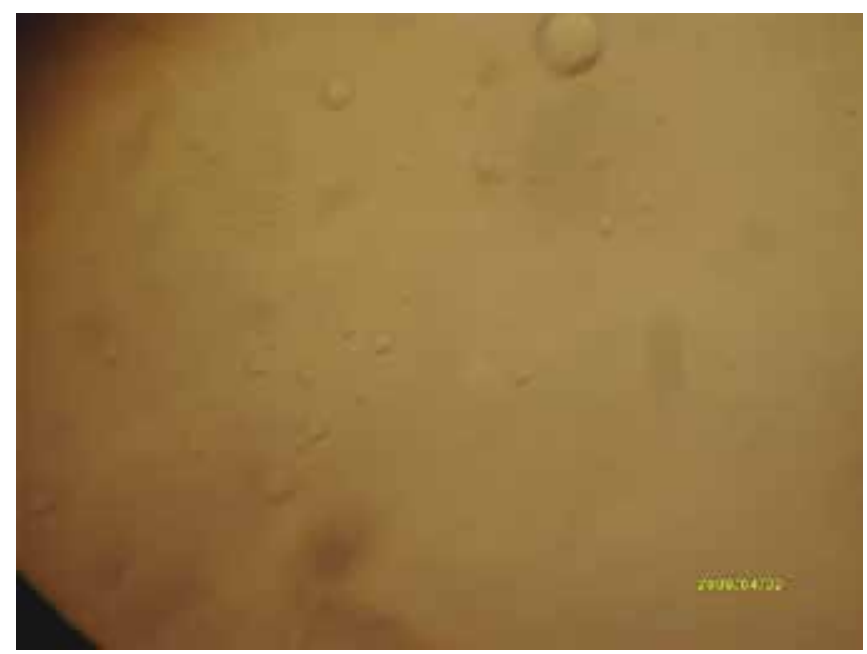

Figura 2. Liposomas hidrosolubles.

\section{Imágenes obtenidas en el microscopio:}

El uso de diferentes excipientes como el etanol y el cloroformo también fue relevante durante este estudio, ya que los liposomas que se utilizaron en los dos geles fueron los obtenidos con el etanol; el cloroformo fue más complicado de usar durante el proceso y el resultado fue nulo.

Con respecto a los conservadores, sólo se observó una optimización en el tiempo, ya que el benzoato de sodio se disolvió con más rapidez que los párabenos, en los cuales se tuvo que aplicar calor en baño María para acelerar su incorporación.

\section{Característica de los extractos}

Los extractos utilizados presentaron las siguientes características:

\begin{tabular}{lll}
\hline Materia prima & Color & $\mathrm{pH}$ \\
\hline Alcohol de amapola & Café & 6 \\
Alcohol de marihuana & Verde & 5 \\
\hline
\end{tabular}

\section{Características organolépticas del producto.}

El producto presenta las siguientes características:

\begin{tabular}{ll}
\hline \multicolumn{1}{c}{ Color } & Paja \\
\hline Olor & Ligeramente alcohol \\
Sabor & ------------ \\
\hline
\end{tabular}

El producto farmacéutico no presentó crecimientos de hongos; no contiene partículas o pirógenos que la puedan contaminar y desestabilizar; presenta un $\mathrm{pH}$ de $8 .{ }^{8}$

Los valores de $\mathrm{pH}$ de los extractos se encuentran dentro del rango de $\mathrm{pH}$ compatible con la piel humana. La formulación final tiene un $\mathrm{pH}$ adecuado para aplicación tópica, lo que nos indica que no producirá daño sobre el lugar de aplicación. Debido a la naturaleza de los extractos antes mencionados, la elaboración del producto nos llevó a obtener el color que presenta dicha formulación, observando que el tono es agradable para la vista del consumidor.

\section{CONCLUSIÓN}

En la elaboración de una forma farmacéutica en gel liposomal con los extractos de marihuana y amapola se obtiene 
un mayor rendimiento en liposomas liposolubles que en liposomas hidrosolubles; por lo tanto, este método se considera una opción adecuada para el desarrollo de la forma farmacéutica en gel.

El uso de diversos excipientes fue importante ya que se pueden mejorar los tiempos de elaboración y costos, donde el etanol brindó mejor rendimiento que el cloroformo, al igual que el benzoato, el cual se disolvió inmediatamente, a diferencia de los párabenos donde se empleó más tiempo para su disolución.

$\mathrm{El} \mathrm{pH}$ de los extractos de productos naturales utilizados y del producto terminado permite que éste sea utilizado adecuadamente para uso tópico.

Los productos naturales seleccionados en combinación para la elaboración de la formulación presentan acción biológica como excelentes analgésicos para problemas musculares, contusiones, tratamiento del reumatismo, etc.

El presente producto comprende un gel para uso tópico; esta composición farmacéutica es un reservorio de ingredientes activos en un sistema de liberación transdérmico presentando una notable estabilidad y características organolépticas agradables.

\section{BIBLIOGRAFÍA}

1. Alfonso R. Gennaro Remington Farmacia. 20a Edición Ed. Panamericana. 2000; II: 1716-1718.

2. Ball E. Liposomas en dermatología. Derm Venez 1995; 33: 15-23.
3. Escuela Andaluza de Salud Pública Glucosamina en Artrosis: ¿previene la progresión de la enfermedad? Boletín Terapéutico Andaluz (BTA) 2002; 18(5): 17-18/1.

4. Escuela Andaluza de Salud Pública. Tratamiento de la artritis reumatoide en adultos. Boletín Terapéutico Andaluz (BTA) 2003; 19 (4): 13-16.

5. Flores J. Farmacología humana. $9^{a}$ Edición Editorial McGraw-Hill Interamericana.

6. Fontelles M. Fármacos y fisiopatología.

7. Frezard. Liposomes: from biophysics to the design of peptide vaccines. Braz J Med Biol Res 1999; 32: 181-189.

8. Gregodiaris G. Liposome Technology: Interactions of liposomes with the biological milieu. Healthcare 2007.

9. Goodman y Gilman. Las bases farmacológicas de la terapéutica. 10 Edición. México. D.F., México. 2002.

10. Isselbacher KJ, Braunwald BE, Harrison WJ. Principios de medicina interna. $13^{\text {va }}$ Ed. Madrid: Interamericana McGraw-Hill 1994: I.

11. Kemp S, Holloword T, Joanne H. Sensory evaluation. Ed John Wiley \& Sons, England 2009.

12. Lista amarilla de estupefacientes. www.incb.org/pdf/s/list/amarilla.pdf. Consulta de marzo de 2010.

13. Naveros CB. Sistemas de transporte y libración de fármacos de aplicación tópica: liposomas multilaminares portadores de acetonido de triamicinolona. Universidad de Granda, 2003.

14. New, "Liposomes-a practical approach," edited by BDHD Rickwood, Vol. 58 (IRL Press at Oxford University Press, 1994).

15. O'Brien T, Welsh J, Dunn FG. ABC of palliative care: Non-malignant conditions. BMJ 1998; 316: 286-289.

16. O’Brien T, Kelly M, Saunders CM. Motor neurone disease: a hospice perspective. BMJ 1992; 304: 471-473.

17. Schäfer $\mathrm{M}, \mathrm{Carter} \mathrm{L}$, Stein C. Interleukin $1 \beta$ and corticotrofin-releasing factor inhibit pain by releasing opioids from immune cells in inflamed tissue. Proc Natl Acad Sci USA 1994; 91: 4219-4223.

18. Wilson JI, Carmody JJ, Walker JS. The importance of the hypothalamohypophyseal-adrenal axis to the anti-inflammatory actions of the k-opioid agonist PNU-50,488H in rats with adjuvant arthritis. J Pharmacol Exp Ther 2000; 294 (3): 1131-1136. 